\title{
Heat Generation/Absorption Effect on Natural Convection Flow in a Vertical Annulus with Time-Periodic Boundary Conditions
}

\author{
Michael O. Oni and Basant K. Jha \\ Department of Mathematics, Ahmadu Bello University, Zaria, Nigeria
}

\author{
Article history \\ Received: 08-04-2019 \\ Revised: 29-04-2019 \\ Accepted: 25-07-2019 \\ Corresponding Author: \\ Michael O. Oni \\ Department of Mathematics, \\ Ahmadu Bello University, \\ Zaria, Nigeria \\ E-mail: michaeloni29@yahoo.com
}

\begin{abstract}
The role of heat generation/absorption on natural convection flow formation in a vertical annulus with prescribed time-periodic heating is investigated in this article. The governing continuity, momentum and energy equations are converted into ordinary differential equation using suitable transformations and closed form expressions are obtained for temperature distribution, velocity profile, Nusselt number, skin friction and mass flow rate in terms of Bessel's function and modified Bessel's function of first and second kinds. Based on depicted line and contour graphs, results show that the inclusion of heat generation/absorption parameter removes the singularity point in periodic velocity and skin friction for unity Prandtl number. In addition, heat generation/absorption parameter respectively increase/decrease periodic temperature distribution, velocity profile, Nusselt number and skin-friction at the surfaces of the cylinders.
\end{abstract}

Keywords: Natural Convection Flow, Fully Developed Region, Heat Generation/Absorption, Time-Periodic Heating, Vertical Annulus

\section{Introduction}

Thermal response in transient heating/cooling problems, such as engine cooling, or how fast a car heats up in the sun and how is it affected by the shape of the windshield has been widely studied. Over the years, a lot of attentions have been drawn to modelling fluid behaviour in a channel or a cylindrical geometry with periodic surface temperature. This can be liken to its everyday application in censor devices and electronics. Steady periodic heating is a vital experimental procedure for measurement of thermal properties. In this method, the thermal properties are realized from a systematic comparison between the temperature and a detailed thermal model.

Steady-periodic flow formation and heat transfer in cylinders is important in several engineering applications including annular fins and in devices undergoing periodic thermal contact such as engine exhaust valves (Aziz and Sofrata, 1981; Houghton et al., 1992; Wu et al., 1996; Paradis et al., 2002). The study of natural convection flow in a channel has been extensively studied for the past five decades (Chung and Anderson, 1961; Yang et al., 1974; Bar-Cohen and Rohsenow, 1984). Wang (1988) examined the free convection flow between vertical plates with periodic heat input. He obtained solution for the case of periodic prescribed temperature as well as prescribed head flux. He resolved that the role of Strouhal number is to reduce the periodic temperature distribution and velocity of the fluid. Since then, a lot of research articles have been published in this field (Jha and Ajibade, 2010; Adesanya et al., 2015; Jha and Ajibade, 2012) by changing his work to the case where the channel is porous (Jha and Ajibade, 2010) with external applied transverse magnetic field (Adesanya et al., 2015) and viscous dissipation effect on flow formation (Jha and Ajibade, 2012).

On the other hand, the importance of heat generation/absorption in moving fluids is of great significance in view of numerous physical applications such as those dealing with chemical reactions and those concerned with dissociating fluids. Different mathematical models have been rendered for internal heat generation effect: Inman (1962) and Ostrach (1952) assumed it to be constant while Chambre (1957) and Toor (1958) pictured it as a function of space. Heat generation is assumed to be the frictional heating and expansion effects of the working fluid based on the works of Gee and Lyon (1957), Modejski (1963) and Toor (1956), while (Moalem, 1976) considered heat source as an inversely proportional value to $(a+b T)$ Later, (Foraboschi and Federico, 1964) gave the volumetric rate of heat generation which is obtained to be directly proportional to $\left(T-T_{0}\right)$ and disclosed that it is an approximation of the state of some exothermic process with $T_{0}$ as the initial temperature. 
Jha and Ajibade (2009) investigated the transient natural convection flow of heat generating/absorbing fluid between vertical porous plates with periodic heat input. They established that, the effect of heat sink is inhibited by large suction value and the impact of suction/injection is suppressed by large value of heat sink. In other related work, (Adesanya, 2015) scrutinized the free convective flow of heat generating fluid through a porous vertical channel with slip velocity and jump temperature. He established that increase in slip and temperature jump parameters increases the periodic flow velocity and fluid temperature respectively.

On studies of periodic heat input in cylindrical geometry, (Cole and Crittenden, 2009) examined the steady-Periodic heating of cylinder by presenting the solutions in term of Green's function. Wu et al. (1996) investigated the transient heat transfer in annular fins of various shapes with their bases subjected to a heat flux changing as a sinusoidal time function. Of recent, (Jha and Oni, 2016; 2019; 2018) obtained an analytical solution for natural convection flow in a tube and an annulus and micro annulus respectively subjected to time-periodic heating. They showed that the skin friction as well as Nusselt number decrease with increase in Strouhal number.

In view of these literature, it is therefore significant to study flow formation and heat transfer of natural convection flow of heat generating/absorbing fluid in a vertical annulus with time-periodic heat input. Thus, the aim of this research work is to examine the impact of heat generation/absorption effect on natural convection flow in a vertical annulus. The governing equations are solved analytically and line and contour graphs are presented to illustrate the effects of various governing parameters. The solution obtained for heat transfer and skin-friction at the surfaces of the annulus can help in minimization of rusting and corrosion.

\section{Mathematical Analysis}

Let us consider a natural convection flow of viscous incompressible heat generating/absorbing fluid in a vertical annulus subjected to periodic heating of the surfaces of the cylinders as shown in Figure 1. The flow is presumed to be laminar and fully developed so that flow motion at the inlet and outlet are neglected. The $r$-axis is the axis in radial direction while the $z$-axis is the axial coordinate which is parallel to the gravitational acceleration $g$ but in opposite direction. The radiuses of the inner and outer cylinders are respectively $a$ and $b$. The surfaces of the cylinders are assumed to be heated periodically to a temperature $\left(T_{1}+T_{2}\right.$ $\cos (\omega t))$ greater than that of initial fluid temperature $T_{0}$. The parameter governing the heat generation/absorption effect is given by $Q=Q_{0}\left(T-T_{0}\right)$ (Foraboschi and Federico, 1964). For the present physical situation and using the Boussinesq's approximation, the governing the continuity, momentum and energy equations in dimensional forms are respectively:

$\frac{\partial u}{\partial z}=0$

$\frac{\partial u}{\partial t}=v\left[\frac{1}{r} \frac{\partial}{\partial r}\left(r \frac{\partial u}{\partial r}\right)\right]+g \beta\left(T-T_{0}\right)$

$\frac{\partial T}{\partial t}=\alpha\left[\frac{1}{r} \frac{\partial}{\partial r}\left(r \frac{\partial T}{\partial r}\right)\right] \pm \frac{Q_{0}\left(T-T_{0}\right)}{\rho C_{p}}$

subject to the following boundary conditions:

$$
\begin{aligned}
& t \leq 0 ; \quad u=0 \quad T=0 \quad \text { for } a \leq r \leq b \\
& t \geq 0\left\{\begin{array}{lll}
u=0 & T=T_{1}+T_{2} \cos (\omega t) & r=a \\
u=0 & T=T_{1}+T_{2} \cos (\omega t) & r=b
\end{array}\right.
\end{aligned}
$$

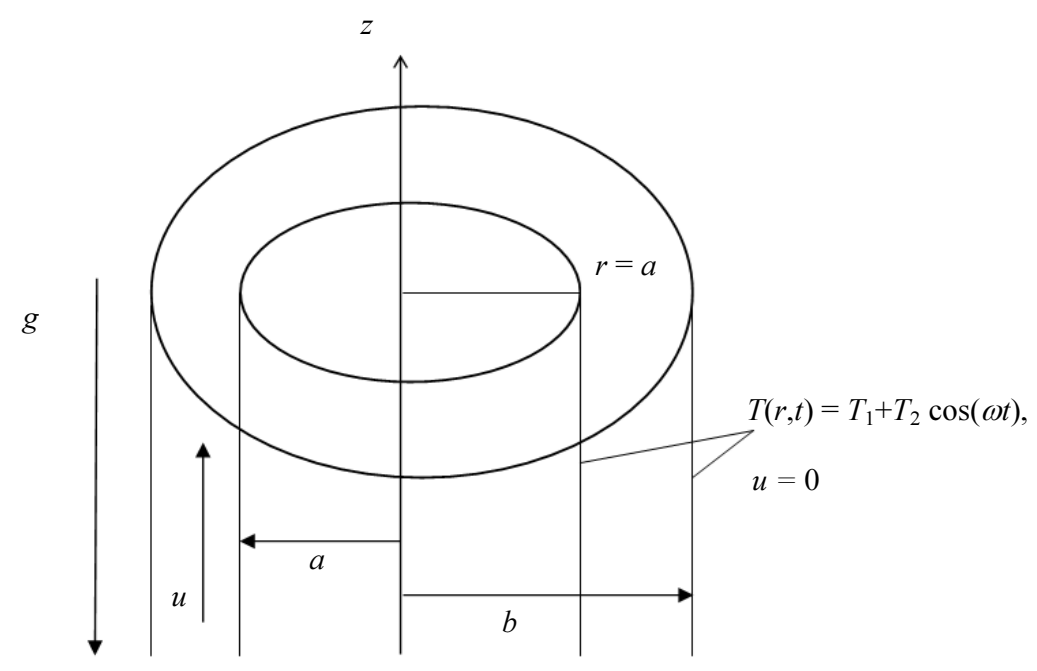

Fig. 1: Schematic diagram of the problem 
To obtain a closed form solution for equations (1-3), the following expressions are used to separate the velocity and temperature into steady and periodic parts (Paradis et al. (2002)):

$$
\begin{aligned}
& u(r, t)=\frac{g \beta a^{2}}{v}\left[\left(T_{1}-T_{0}\right) A(R)+T_{2} B(R) e^{i \omega t}\right] \\
& T(r, t)=T_{0}+\left[\left(T_{1}-T_{0}\right) F(R)+T_{2} G(R) e^{i \omega t}\right]
\end{aligned}
$$

where, $A(R), F(R)$ represents the steady parts and $B(R)$, $G(R)$ represent the unsteady parts of the velocity and temperature respectively.

On substituting equations (5) and (6) into the momentum and energy equations (2) and (3) respectively, we obtain the following dimensionless ordinary differential equations:

$$
\begin{aligned}
& \frac{d^{2} A(R)}{d R^{2}}+\frac{1}{R} \frac{d A(R)}{d R}=-F(R) \\
& \frac{d^{2} B(R)}{d R^{2}}+\frac{1}{R} \frac{d B(R)}{d R}-i S t B(R)=-G(R) \\
& \frac{d^{2} F(R)}{d R^{2}}+\frac{1}{R} \frac{d F(R)}{d R} \pm H^{2} F(R)=0 \\
& \frac{d^{2} G(R)}{d R^{2}}+\frac{1}{R} \frac{d G(R)}{d R}-\left(i S t \operatorname{Pr} \mp H^{2}\right) G(R)=0 \\
& F(R)=\frac{\left[Y_{0}(\lambda H)-Y_{0}(H)\right] J_{0}(H R)+\left[J_{0}(H)-J_{0}(\lambda H)\right] Y_{0}(H R)}{\left[J_{0}(H) Y_{0}(\lambda H)-Y_{0}(H) J_{0}(\lambda H)\right]} \\
& G(R)=\frac{\left[Y_{0}\left(\lambda M_{1}\right)-Y_{0}\left(M_{1}\right)\right] J_{0}\left(M_{1} R\right)+\left[J_{0}\left(M_{1}\right)-J_{0}\left(\lambda M_{1}\right)\right] Y_{0}\left(M_{1} R\right)}{\left[J_{0}\left(M_{1}\right) Y_{0}\left(\lambda M_{1}\right)-Y_{0}\left(M_{1}\right) J_{0}\left(\lambda M_{1}\right)\right]} \\
& B(R)=C_{13} I_{0}(R \sqrt{i S t})+C_{14} K_{0}(R \sqrt{i S t})+ \\
& \frac{\left[Y_{0}\left(\lambda M_{1}\right)-Y_{0}\left(M_{1}\right)\right] J_{0}\left(M_{1} R\right)+\left[J_{0}\left(M_{1}\right)-J_{0}\left(\lambda M_{1}\right)\right] Y_{0}\left(M_{1} R\right)}{\left(H^{2}+i S t(1-\operatorname{Pr})\right)\left[J_{0}\left(M_{1}\right) Y_{0}\left(\lambda M_{1}\right)-Y_{0}\left(M_{1}\right) J_{0} \lambda M_{1}\right]} \\
& A(R)=\frac{1}{H^{2}} \frac{\left[Y_{0}(\lambda H)-Y_{0}(H)\right] J_{0}(H R)+\left[J_{0}(H)-J_{0}((\lambda H)] Y_{0}(H R)\right.}{\left[J_{0}(H) Y_{0}(\lambda H)-Y_{0}(H) J_{0}(\lambda H)\right]}+C_{11} \ln (R)+C_{12}
\end{aligned}
$$

where, $S t$ is the Strouhal number which is proportional to the frequency of periodic heating and inversely proportional to kinematic viscosity, $P r$ is the Prandtl number which is directly proportional to kinematic viscosity and inversely proportional to thermal

\section{Heat Generating Case}

The solution of equation (7) with positive sign of $\mathrm{H}^{2}$ along with boundary conditions (8) are given below as:

The skin-friction $(\tau)$ at the surfaces of the cylinders is obtained by differentiating the unsteady velocity (Yang et al., 1974):

$$
\begin{gathered}
\tau_{1}=\left.\frac{d B_{R}}{d R}\right|_{R=1}=\sqrt{i S t}\left[C_{13} I_{1}(\sqrt{i S t})-C_{14} K_{1}(\sqrt{i S t})\right]-\frac{M_{1}\left[\left(C_{7} Y_{1}\left(M_{1}\right)+C_{8} Y_{1}\left(M_{1}\right)\right)\right]}{\left(H^{2}+i S t(1-\operatorname{Pr})\right)} \\
\tau_{\lambda}=-\left.\frac{d B_{R}}{d R}\right|_{R=\lambda}=\sqrt{i S t}\left[C_{14} K_{1}(\lambda \sqrt{i S t})-C_{13} I_{1}(\lambda \sqrt{i S t})\right]+\frac{M_{1}\left[\left(C_{7} J_{1}\left(\lambda M_{1}\right)+Y_{1}\left(\lambda M_{1}\right)\right)\right]}{\left(H^{2}+i S t(1-\operatorname{Pr})\right)}
\end{gathered}
$$


The rate of heat transfer between the surfaces of the cylinders and the fluid is obtained by differentiating the unsteady temperature as follows:

$$
\begin{aligned}
& \begin{array}{ll}
N u_{1}=-\left.\frac{d G_{R}}{d R}\right|_{R=1}=M_{1}\left(C_{7} J_{1}\left(M_{1}\right)+C_{8} Y_{1}\left(M_{1}\right)\right) & \text { Heat Absorbing Case } \\
N u_{\lambda}=-\left.\frac{d G_{R}}{d R}\right|_{R=\lambda}=-M_{1}\left(C_{7} J_{1}\left(\lambda M_{1}\right)+C_{8} Y_{1}\left(\lambda M_{1}\right)\right) & \begin{array}{c}
\text { In similar manner as for heat } \\
\text { solution of equation (7) with negativ } \\
\text { with boundary conditions (8) are give }
\end{array} \\
F(R)=\frac{\left[K_{0}(\lambda H)-K_{0}(H)\right] I_{0}(H R)+\left[I_{0}(H)-I_{0}(\lambda H)\right] K_{0}(H R)}{\left[I_{0}(H) K_{0}\left(\lambda H-K_{0}(H) I_{0}(\lambda H)\right]\right.} & {\left[I_{0}\left(M_{2}\right) K_{0}\left(\lambda M_{2}\right)-K_{0}\left(M_{2}\right) I_{0}\left(\lambda M_{2}\right)\right]}
\end{array} \\
& \left.G(R)=\frac{\left[K_{0}\left(\lambda M_{2}\right)-K_{0}\left(M_{2}\right)\right] I_{0}\left(M_{2} R\right)+\left[I_{0}\left(M_{2}\right)-I_{0}\left(\lambda M_{2}\right)\right] K_{0}\left(M_{2} R\right)}{\left[I_{0}(H) K_{0}(\lambda H)-K_{0}(H) I_{0}(\lambda H)\right]}\right] \\
& B(R)=C_{15} I_{0}(R \sqrt{i S t})+C_{16} K_{0}(R \sqrt{i S t})-\frac{\left[K_{0}\left(\lambda M_{2}\right)-K_{0}\left(M_{2}\right)\right] I_{0}\left(M_{2} R\right)+\left[I_{0}\left(M_{2}\right)-I_{0}\left(\lambda M_{2}\right)\right] K_{0}\left(M_{2} R\right)}{\left(H^{2}+i S t(\operatorname{Pr}-1)\right)\left[I_{0}\left(M_{2}\right) K_{0}\left(\lambda M_{2}\right)-K_{0}\left(M_{2}\right) I_{0}\left(\lambda M_{2}\right)\right]} \\
& A(R)=C_{9} \ln (R)+C_{10}-\frac{1}{H^{2}}-\left[\frac{\left.\left[K_{0}(\lambda H)-K_{0}(H)\right] I_{0}(H R)+\left[I_{0}(H)-I_{0}(\lambda H)\right] K_{0}(H R)\right]}{[}\right]
\end{aligned}
$$

Also, the skin-friction $(\tau)$ at the surfaces of the cylinders is obtained by differentiating the unsteady velocity (Yang et al., 1974):

$$
\begin{aligned}
& \tau_{1}=\left.\frac{d B_{R}}{d R}\right|_{R=1}=\sqrt{i S t}\left[C_{15} I_{1}(\sqrt{i S t})-C_{16} K_{1}(\sqrt{i S t})\right]-\frac{M_{2}\left[\left(C_{5} I_{1}\left(M_{2}\right)-C_{6} K_{1}\left(M_{2}\right)\right)\right]}{\left(H^{2}+i S t(\operatorname{Pr}-1)\right)} \\
& \tau_{\lambda}=-\left.\frac{d B_{R}}{d R}\right|_{R=\lambda}=\sqrt{i S t}\left[C_{16} K_{1}(\lambda \sqrt{i S t})-C_{15} I_{1}(\lambda \sqrt{i S t})\right]+\frac{M_{2}\left[\left(C_{5} I_{1}\left(\lambda M_{2}\right)-C_{6} K_{1}\left(\lambda M_{2}\right)\right)\right]}{\left(H^{2}+i S t(\operatorname{Pr}-1)\right)}
\end{aligned}
$$

The Nusselt number which measures the rate of heat transfer between the surfaces of the cylinders and the fluid is obtained by taking the first derivative of the periodic temperature distribution as follows:

$$
\begin{aligned}
& N u_{1}=\left.\frac{d G_{R}}{d R}\right|_{R=1}=M_{2}\left(C_{5} I_{1}\left(\lambda M_{2}\right)-C_{6} K_{1}\left(M_{2}\right)\right) \\
& N u_{\lambda}=\left.\frac{d G_{R}}{d R}\right|_{R=1}=M_{2}\left(C_{5} I_{1}\left(\lambda M_{2}\right)-C_{6} K_{1}\left(\lambda M_{2}\right)\right) \\
& M=\int_{1}^{\lambda} R B(R) d R=N_{3}+N_{4}
\end{aligned}
$$

Since the flow is periodic, in order to have a good insight on the problem, the phase as well as amplitude of both temperature and velocity are computed. The phases $\psi$ and $\chi$ which is the angle between the temperatures $G_{i}$ and $G_{R}$ and velocities $B_{i}$ and $B_{R}$ respectively while the amplitudes $|G|$ and $|B|$ denotes the absolute temperature and velocity at any given point in the cylinder.

The phase $(\psi)$ of temperature and phase $(\chi)$ of velocity are obtained by the expression (Chung and Anderson, 1961):

$\psi=\tan ^{-1}\left(\frac{G_{i}}{G_{R}}\right) \quad$ and $\quad \chi=\tan ^{-1}\left(\frac{B_{i}}{B_{R}}\right)$

Also, the amplitude of the unsteady temperature and unsteady velocity are given respectively by:

$|G|=\sqrt{\left(G_{R}{ }^{2}+G_{i}^{2}\right)}$ and $\quad|B|=\sqrt{\left(B_{R}{ }^{2}+B_{i}^{2}\right)}$ 


\section{Results and Discussion}

The solutions obtained are physical presented in terms of line and contour graphs in order to show the effects of governing parameters (Strouhal number $(S t)$, Prandtl number $(P r)$, heat generation/absorption parameter $(\mathrm{H})$ and ratio of radiuses $(\lambda)$. The values of Prandtl number are taken over the range of $0.5 \leq \operatorname{Pr} \leq 2.0$ with a reference value of $P r=0.71$ (atmospheric air). While those of heat generation/absorption parameter have been selected as 0.5 and 2.0. In all depicted graphs, figures with caption (a) represent the heat generating fluid case while those with caption (b) represent heat absorbing fluid case.

It is interesting to note that the steady temperature solution (Equation (10) and (19)) is not unity as in
(Wang, 1988; Jha and Ajibade, 2010; Adesanya, 2015; Jha and Oni, 2016). This can be attribute to the fact that the fluid considered here is heat generating/absorbing.

Figures $2 \mathrm{a}$ and $2 \mathrm{~b}$ depict periodic temperature profile at different values of $S t$ and $H$ for heat generation and absorption cases respectively. It is observed that the role of heat generation is to increase fluid temperature for heat generating fluid case while the reverse trend occurs for heat absorbing fluid case. This is true because increase in heat generation parameter increases the heat generating effect of the fluid and hence enhances fluid temperature. Also, fluid temperature for both cases decreases with increase in Strouhal number $(S t)$.

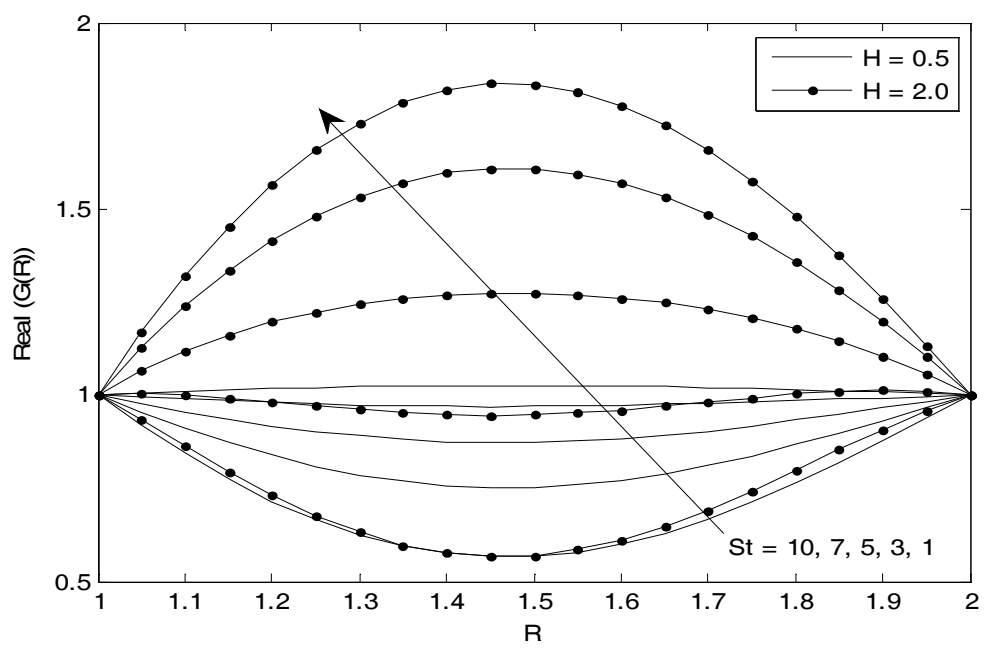

(a)

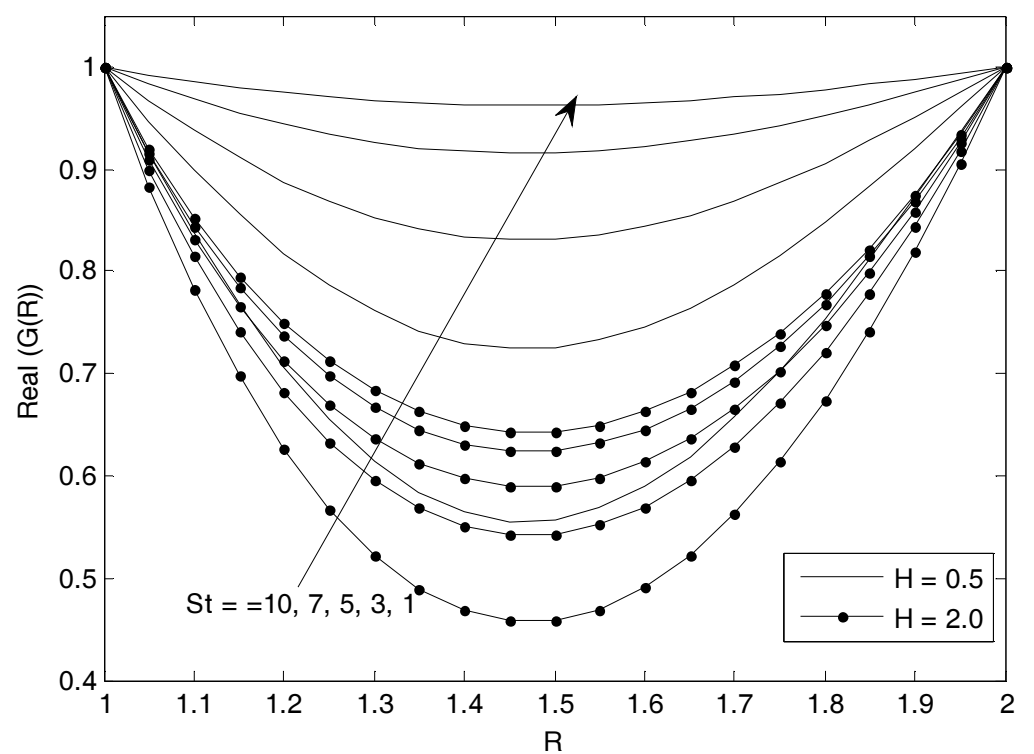

(b)

Fig. 2: (a) Temperature profile for different value of $\mathrm{H}$ an $\mathrm{St}$ at $\mathrm{Pr}=0.71, \lambda=2.0$; (b) Temperature profile for different values of $\mathrm{H}$ and $\mathrm{St}$ at $\mathrm{Pr}=0.71, \lambda=2.0$ 


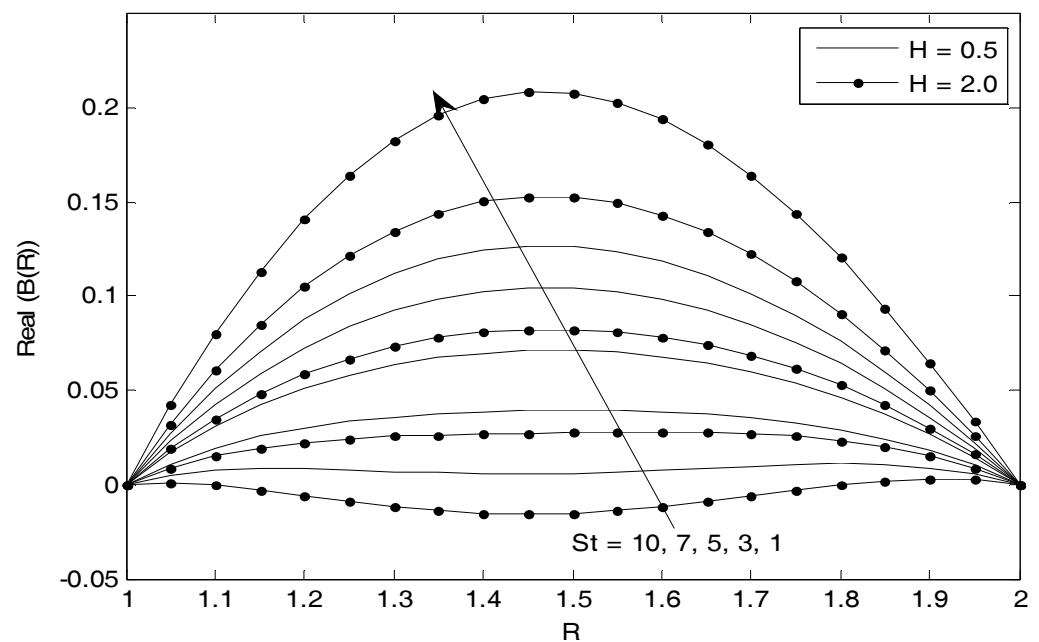

(a)

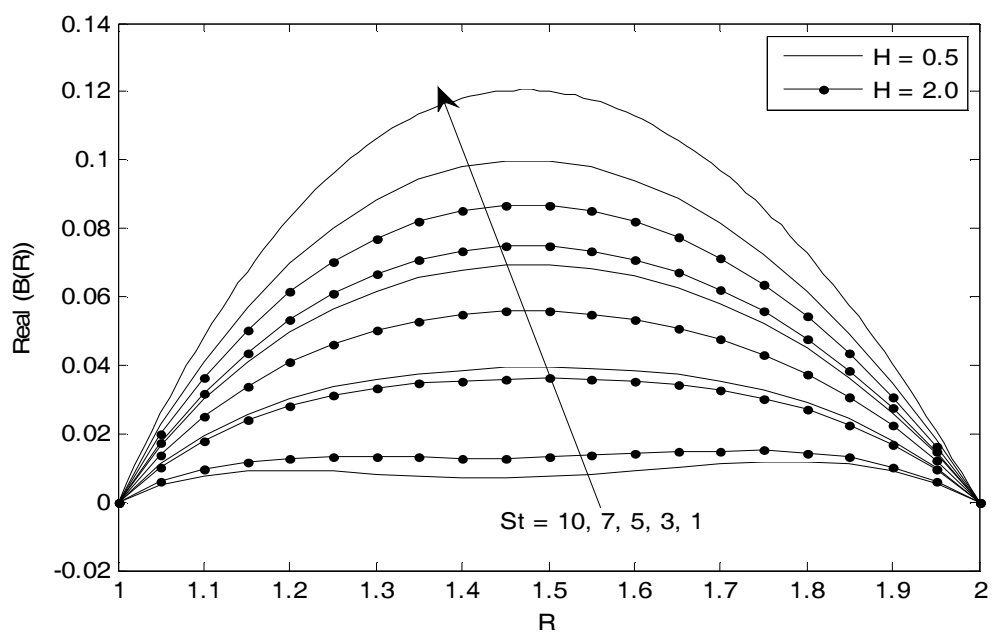

(b)

Fig. 3: (a) Velocity profile for different values of $\mathrm{H}$ and $\mathrm{St}$ at $\mathrm{Pr}=0.71, \lambda=2.0$; (b) velocity profile for different values of $\mathrm{H}$ and $\mathrm{St}$ at $\operatorname{Pr}=0.71, \lambda=2.0$

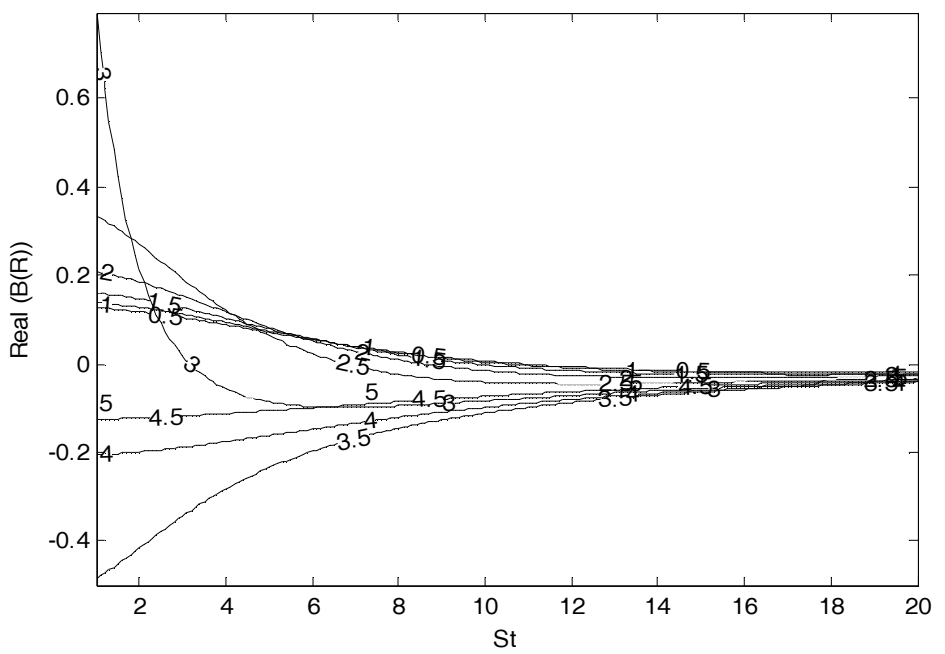

(a) 


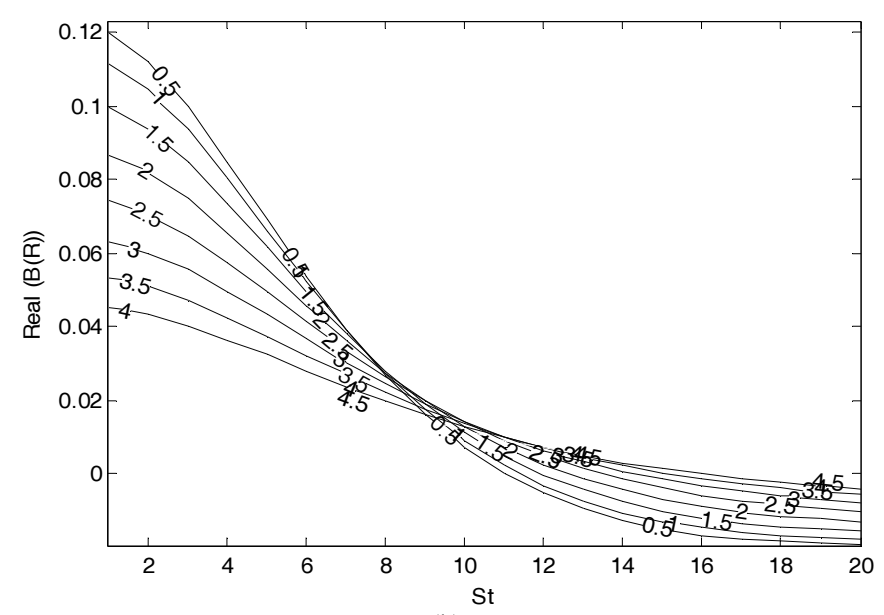

(b)

Fig. 4: (a) velocity profile for different values of $\mathrm{St}$ and $\mathrm{H}$ at $\mathrm{Pr}=0.71, \lambda=2.0(\mathrm{R}=1.5)$ (b) velocity profile for different values of $\mathrm{St}$ and $\mathrm{H}$ at $\mathrm{Pr}=0.71, \lambda=2.0(\mathrm{R}=1.5)$

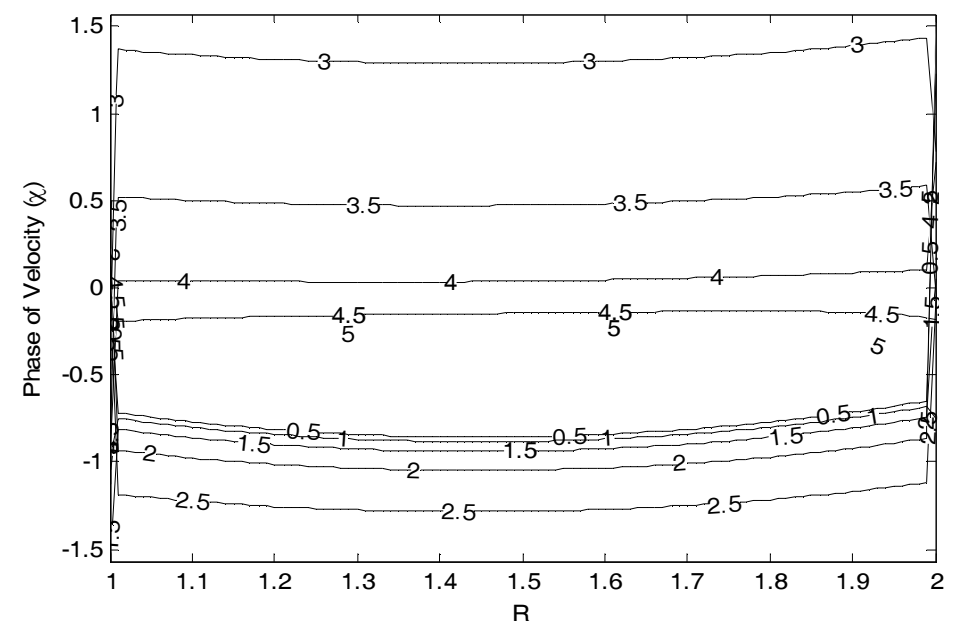

(a)

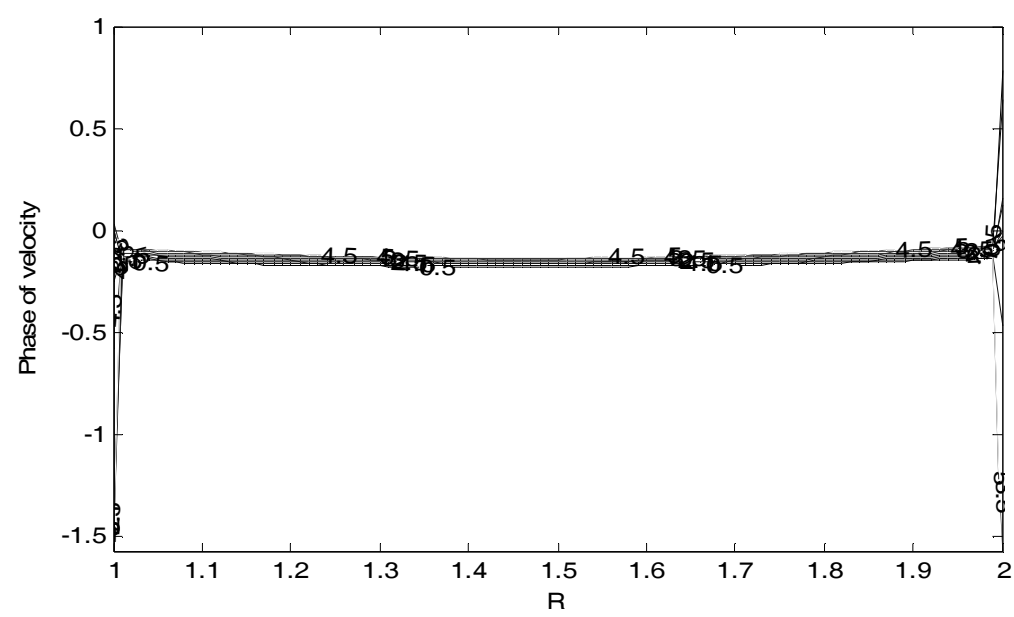

(b)

Fig. 5: (a) Phase of velocity $(B(R))$ for different values of $H$ at $\operatorname{Pr}=0.71, S t=5.0$ and $\lambda=2.0(b)$ Phsdr of velocity $(B(R))$ for different values of $\mathrm{H}$ at $\mathrm{Pr}=0.71, \mathrm{St}=5.0$ and $\lambda=2.0$ 


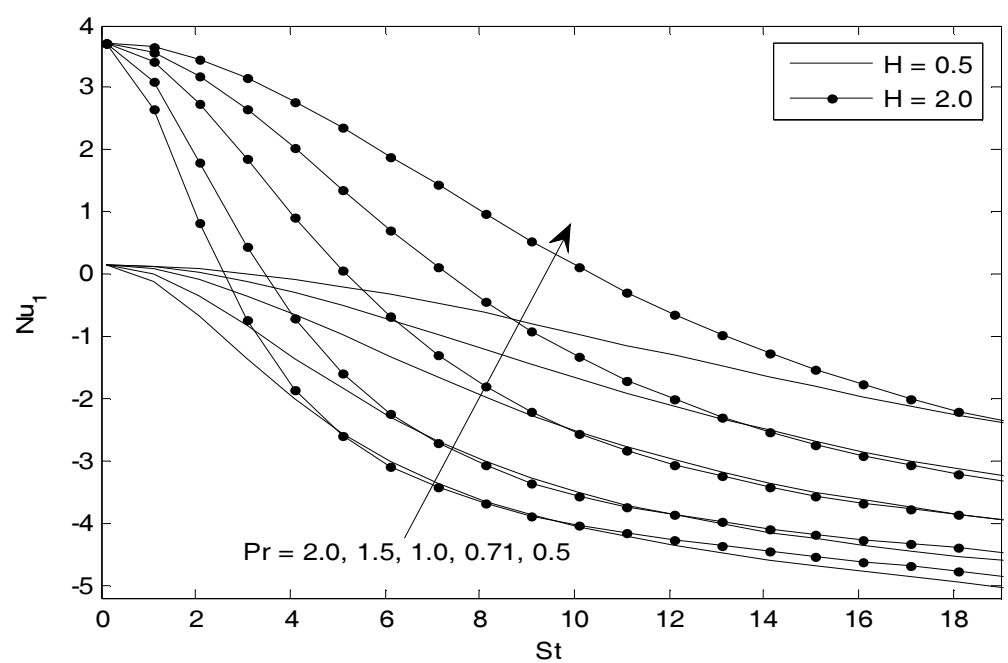

(a)

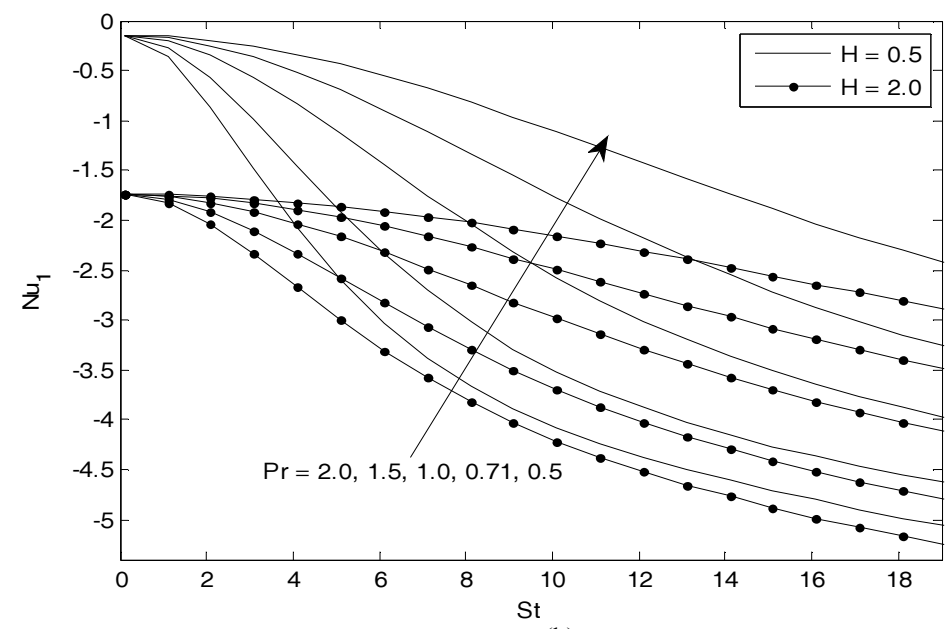

(b)

Fig. 6: (a) Nusselt number for different values of $H, \operatorname{Pr}$ and $\mathrm{St}$ at $\lambda=2.0(\mathrm{R}=1)$ (b) Nusselt number for different values of $\mathrm{H}$, Pr and $\mathrm{St}$ at $\lambda=2.0(\mathrm{R}=1)$

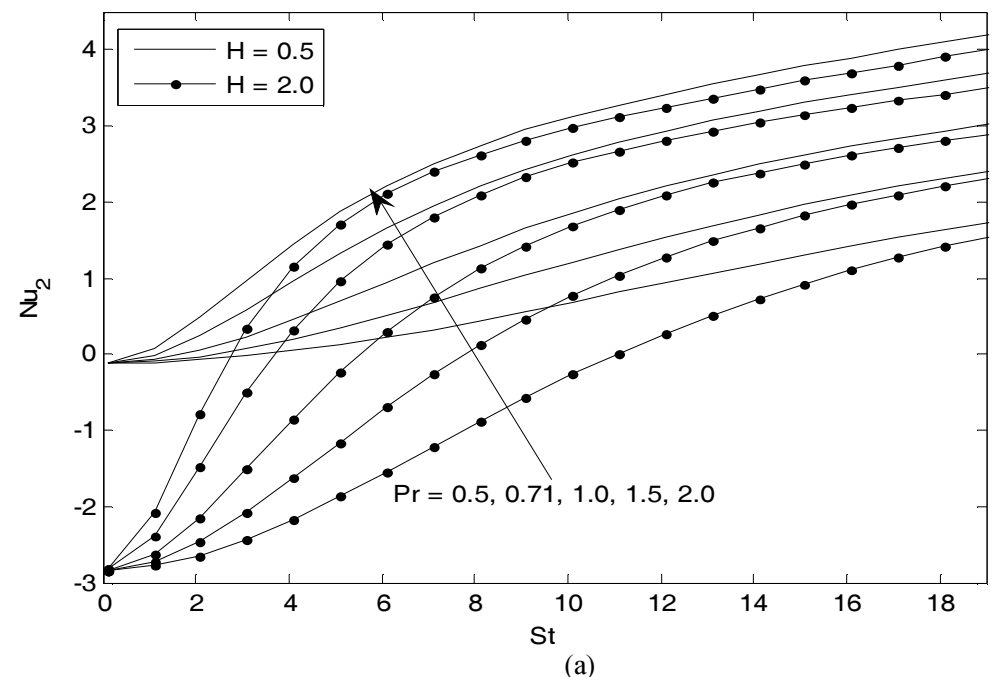




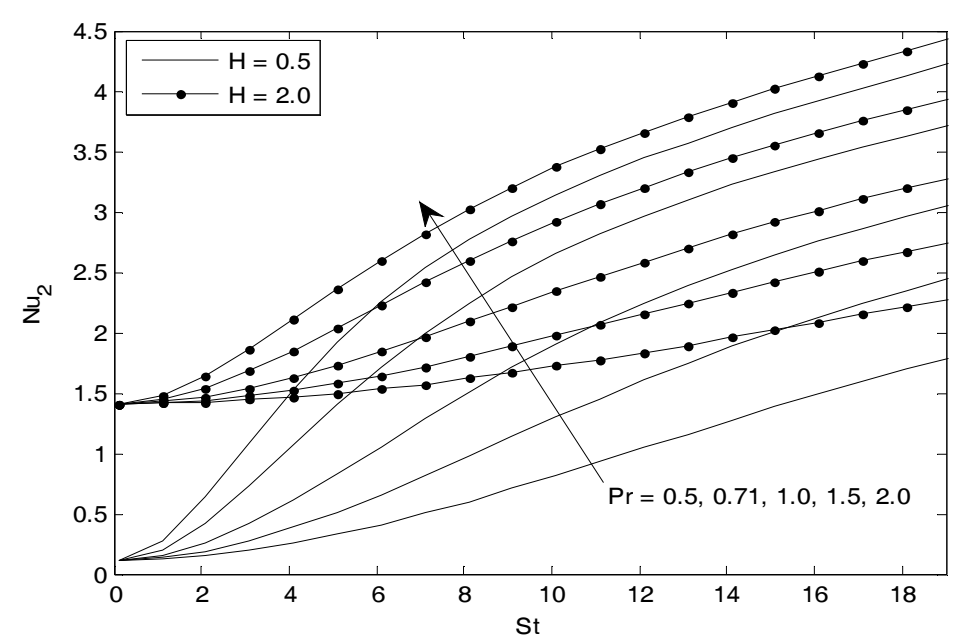

(b)

Fig. 7: (a) Nusselt number for different values of $H, \operatorname{Pr}$ and St at $\lambda=2.0(\mathrm{R}=\lambda)$ (b) Nusselt number for different values of $H$, Pr and St at $\lambda=2.0(\mathrm{R}=\lambda)$

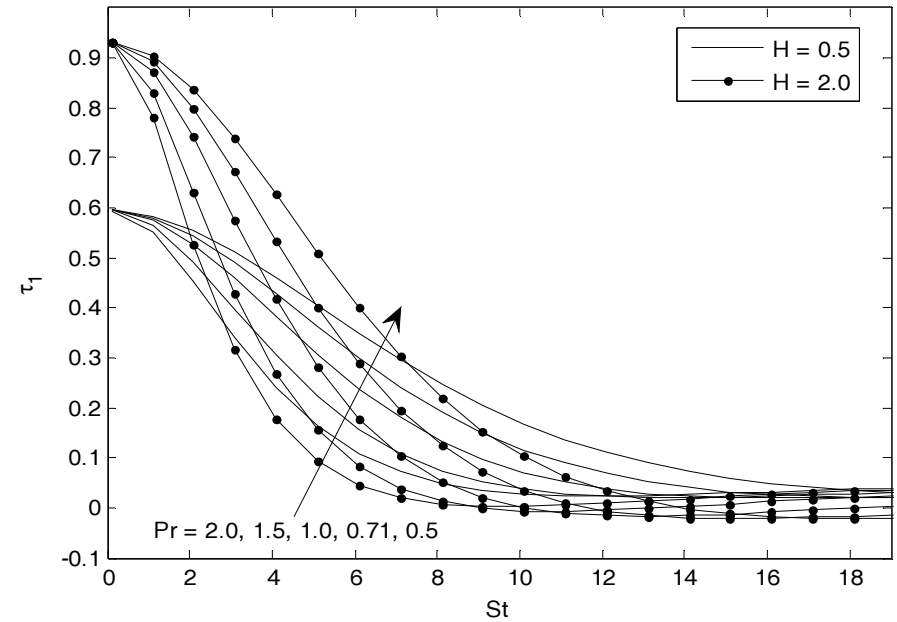

(a)

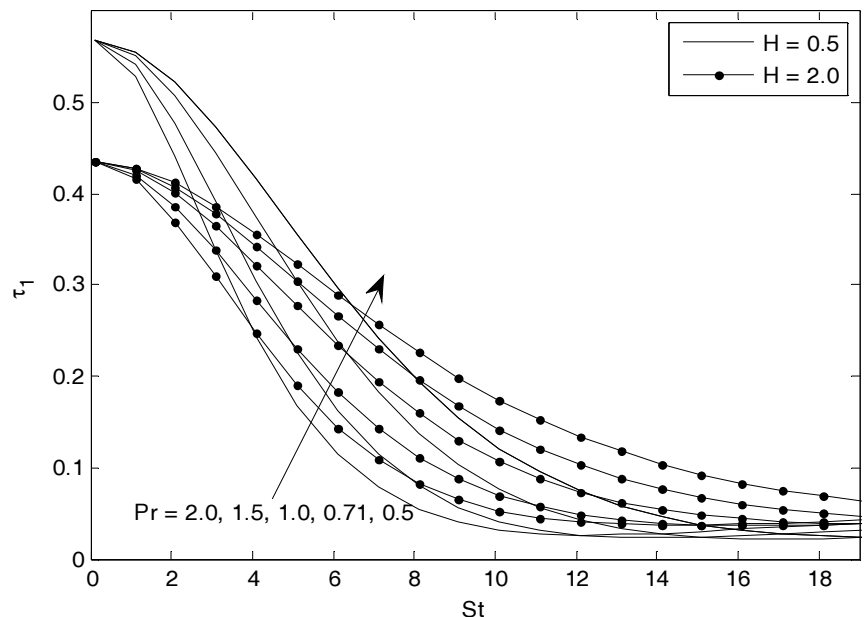

(b)

Fig. 8: (a) Nusselt number for different values of $H, \operatorname{Pr}$ and $\mathrm{St}$ at $\lambda=2.0(\mathrm{R}=\lambda)$ (b) Nusselt number for different values of $\mathrm{H}$, Pr and St at $\lambda=2.0(\mathrm{R}=\lambda)$ 


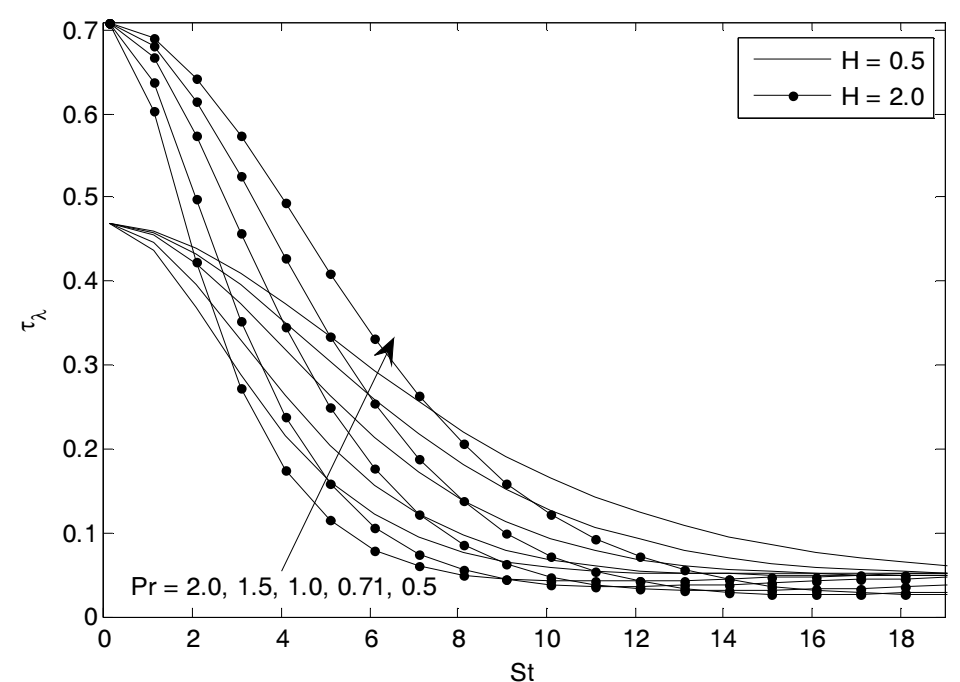

(a)

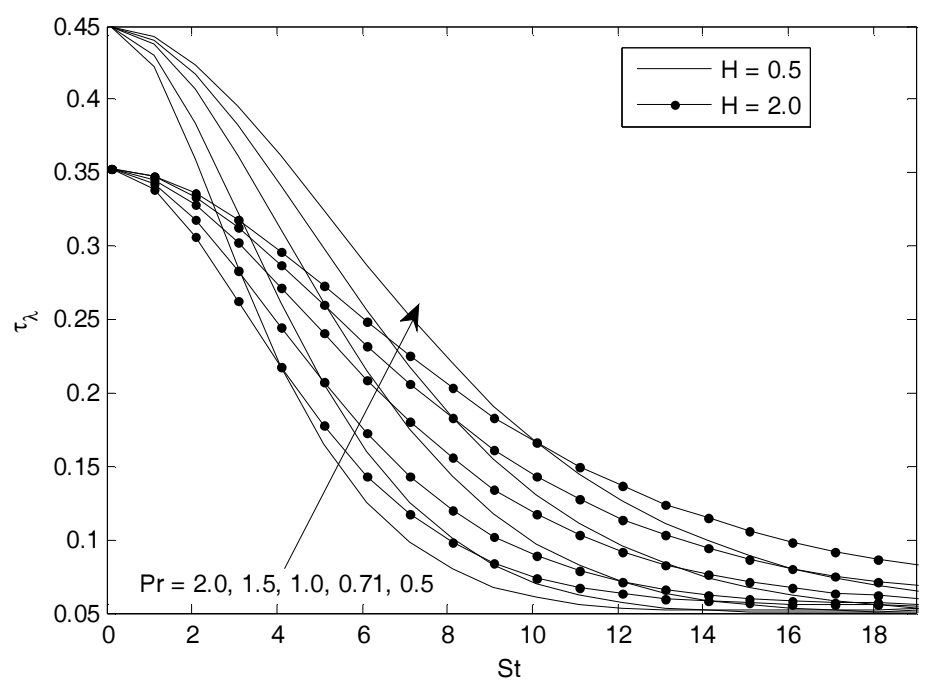

(b)

Fig. 9: (a) Skin friction for different values of $H, P r$ and $\mathrm{St}$ at $\lambda=2.0(\mathrm{R}=\lambda)$ (b) Skin friction for different values of $\mathrm{H}$, Pr and $\mathrm{St}$ at $\lambda=2.0(\mathrm{R}=\lambda)$

Figure $3 \mathrm{a}$ and $3 \mathrm{~b}$ on the other hand present the combined effect of periodic heating at the surfaces of the cylinders $(S t)$ and heat generation/absorption $(H)$ on periodic flow formation on the annulus for heat generating/absorbing fluids respectively. It is evident from the graphs that periodic velocity decreases with increase in Strouhal number $(S t)$ for both heat generating and absorbing case. This can be explained to the fact that increasing frequency of heating leads to weaker convection with in turn retard flow velocity. Also, heat generation effect increases fluid velocity for heat generating case while the converse is noticed for heat absorbing case. This can be physically understand since heat generation effect increase fluid temperature with will increase the kinematic of the fluid and hence the velocity.
Figure $5 \mathrm{a}$ and $5 \mathrm{~b}$ represent the effect of periodic heating at the surfaces of the cylinders $(S t)$ and heat generation/absorption parameter on phase of periodic velocity for heat generating/absorbing case respectively. It is seen that the angle between the velocities is linear for both case. In addition, the phase of heat absorbing case is independent of hear absorption parameter as well as radial axis.

The effect of periodic heating at the surfaces of the cylinders $(S t)$, heat generation/absorption parameter $(H)$ and Prandtl number $(\mathrm{Pr})$ on rate of heat transfer for periodic part represented by Nusselt number is depicted in Fig. 6a, 7a and 6b, 7b at the outer surface of the inner cylinder and inner surface of the outer cylinder respectively for heat generating/absorbing fluid respectively. It is found that rate of heat transfer at the 
outer surface of the inner cylinder increases with increase in heat generation parameter (Figs. 6a and 7a) and decreases with increase in Strouhal number and Prandtl number, while the reverse case is observed at the inner surface of outer cylinder. Whereas for heat absorbing case, the rate of heat transfer at the outer surface of the inner cylinder decreases with increase in heat absorption parameter, Strouhal number and Prandtl number while is converse is seen for rate of heat transfer at the inner surface of outer cylinder. In addition, for all cases, points of inflexion are noticed for different heat generation/absorption parameter.

Figures $8 \mathrm{a}, 9 \mathrm{a}, 8 \mathrm{~b}$ and $9 \mathrm{~b}$ respectively present the role of periodic heating at the surfaces of the cylinders $(S t)$, heat generation/absorption parameter $(H)$ and Prandtl number $(P r)$ on skin-friction at the outer surface of the inner cylinder and inner surface of the outer cylinder respectively for heat generating/absorbing fluid. It is found that the drag effect for heat generating case at both surfaces of the cylinders (Figs. 8a and 9a) decreases with increase in Strouhal number and Prandtl number but increases with increase on heat generation parameter.

For heat absorbing fluid on the other hand, the role of Strouhal number, heat absorption parameter and Prandtl number is to decrease skin friction at the surfaces of the cylinders. In addition, in both cases, the skin friction is seen to be higher at the outer surface of inner cylinder than at the inner surface of the outer cylinder. Furthermore, for large Strouhal number, the skin friction tends to zero for all cases and is independent of Prandtl number and kind of fluid considered. This physically explains Fig. $4 \mathrm{a}$ and $4 \mathrm{~b}$ since the velocity is constant for large value of $(S t)$, hence its derivative (skin friction) is zero.

\section{Conclusion}

This work investigated the role of heat generation/absorption parameter on natural convection flow in a vertical annulus subject to prescribed periodic heating at the surfaces of the cylinders. The governing continuity, momentum and energy equations are transformed into ordinary differential equation and closed form expressions are obtained for temperature, velocity, Nusselt number, skin friction and mass flow rate in terms of Bessel's function and modified Bessel's function. Based on the results obtained from depicted graphs, the following conclusions can be drawn:

1. It is interesting to note the inclusion of heat generation/absorption effects removes the singularity point that exists at unity $(\mathrm{Pr})$ for periodic velocity and skin friction (Wang, 1988; Jha and Ajibade, 2010; 2015; Jha and Oni, 2016).

2. Also, the steady temperature is seen to be dependent on $H$ and ratio of radiuses $\lambda$ and $R$ as against the well know unity $(F(R)=1)$ (Wang, 1988; Jha and Ajibade, 2010; Adesanya, 2015; Jha and Oni, 2016).

3. Periodic temperature, velocity, Nusselt number and skin friction decrease with increase in Strouhal number

4. Heat generation/absorption parameter respectively increase/decrease temperature, velocity, Nusselt number and skin-friction at the surfaces of the cylinders

\section{Acknowledgment}

The authors are thankful to all reviewers for their positive comments on improving the quality of this article.

\section{Author's Contributions}

Author one solve the problem, plot the graphs and discuss the results. While author two gives the problem formulation.

\section{Conflict of Interest}

The authors declare that there is no conflict of interest.

\section{References}

Adesanya, S.O., 2015. Free convective flow of heat generating fluid through a porous vertical channel with velocity slip and temperature jump. Ain Shams Eng. J., 6: 1045-1052. DOI: 10.1016/j.asej.2014.12.008

Adesanya, S.O., E.O. Oluwadare, J.A. Falade and O.D. Makinde, 2015. Hydromagnetic natural convection flow between vertical parallel plates with time-periodic boundary conditions. J. Magnetism Magnetic Mater., 396: 295-303. DOI: 10.1016/j.jmmm.2015.07.096

Aziz, A. and H. Sofrata, 1981. Fin performance in an oscillating temperature environment. Applied Energy, 9: 13-21. DOI: 10.1016/0306-2619(81)90038-6

Bar-Cohen, A. and W.M. Rohsenow, 1984. Thermally optimum spacing of vertical, natural convection cooled, parallel plates. Trans. ASME J. Heat Transf., 106: 116-123. DOI: 10.1115/1.3246622

Chambre, P.L., 1957. Laminar boundary layer with distributed heat sources or sinks. Applied Sci. Res. A, 6 393-401. DOI: 10.1007/BF03185044

Chung, P.M. and A.D. Anderson, 1961. Unsteady laminar free convection. ASME J. Heat Transfer, 83: 473-478. DOI: $10.1115 / 1.3683670$

Cole, K.D. and P.E. Crittenden, 2009. Steady-Periodic heating of cylinder. J. Heat Transfer, 131: 1-7. DOI: $10.1115 / 1.3139107$

Foraboschi, F.P. and I.D. Federico, 1964. Heat transfer in laminar flow of non-Newtonian heat generating fluids. Int. J. Heat Mass Transfer, 7: 315-318. DOI: 10.1016/0017-9310(64)90107-3 
Gee, R.E. and J.B. Lyon, 1957. Non-isothermal flow of viscous non-newtonian fluids. Ind. Eng. Chem., 49: 956-960. DOI: 10.1021/ie50570a024

Houghton, J.M., D.B. Ingham and P.J. Heggs, 1992. The one-dimensional analysis of oscillatory heat transfer in a fin assembly. ASME J. Heat Transfer, 114: 548-552. DOI: $10.1115 / \mathrm{S} 1.2911316$

Inman, R.M., 1962. Experimental study of temperature distribution in laminar tube flow of a fluid with internal heat generation. Int. J. Heat Mass Transfer, 5: 1053-1053. DOI: 10.1016/0017-9310(62)90058-3

Jha, B.K and A.O. Ajibade, 2012. Effect of viscous dissipation on natural convection flow between vertical parallel plates with time-periodic boundary conditions. Comm. Nonlinear Sci. Numer. Simulat., 17: 1576-1587. DOI: 10.1016/j.cnsns.2011.09.020

Jha, B.K and M.O. Oni, 2018. Natural convection flow in a vertical micro-annulus with time-periodic thermal boundary conditions: An exact solution. Multidiscipline Model. Mater. Structures, 14: 1064-1081. DOI: 10.1108/MMMS-09-2017-0111

Jha, B.K. and A.O. Ajibade, 2010. Free convective flow between vertical porous plates with periodic heat input. ZAMM. Z. Angew. Math. Mech., 90: 185-193. DOI: 10.1002/zamm.200900268

Jha, B.K. and A.O. Ajibade, 2009. Unsteady free convection Couotte flow of heat generating/absorbing fluids between vertical porous plates with periodic heat input. Int. Communi. Heat Mass Transfer, 36: 624-631. DOI: 10.1016/j.icheatmasstransfer.2009.03.003

Jha, B.K. and M.O. Oni, 2016. Natural convection flow in a vertical tube inspired by time-periodic heating. Alexandria Eng. J., 55: 3145-3151. DOI: 10.1016/J.AEJ.2016.08.025

Jha, B.K. and M.O. Oni, 2019. Natural convection flow in a vertical annulus with time-periodic thermal boundary conditions. Propulsion Power Res., 8: 47-55. DOI: 10.1016/j.jppr.2018.12.002.

Moalem, D., 1976. Steady state heat transfer with porous medium with temperature dependent heat generation. Int. J. Heat Mass Transfer, 19: 529-537. DOI: 10.1016/0017-9310(76)90166-6

Modejski, J., 1963. Temperature distribution in channel flow with friction, Int. J. Heat Mass Transfer, 6: 49-51. DOI: 10.1016/0017-9310(63)90028-0

Ostrach, S., 1952. Laminar natural convection flow and heat transfer of fluids with and without heat sources in channels with constant wall temperatures. NACA TN.

Ostrach, S., 1957. Combined natural- and forcedconvection flow and heat transfer of fluids with and without heat sources in channels with linearly varying wall temperatures. NACA TN.
Paradis, I., J.R. Wagner and E.E. Marotta, 2002. Thermal periodic contact of exhaust valves in sparkignition air-cooled engines. J. Thermophys. Heat Transfer, 16: 356-365. DOI: 10.2514/2.6712

Toor, H.L., 1956. The energy equation for viscous flow. Ind. Eng. Chem., 48: 922-926. DOI: $10.1021 /$ ie $50557 \mathrm{a} 035$

Toor, H.L., 1958. Heat transfer in forced convection with internal heat generation. J. Am. Inst. Chem. Eng., 4: 319- 323. DOI: 10.1002/aic.690040317

Wang, C.Y., 1988. Free convection between vertical plates with periodic heat input. Trans. ASME J., Heat Transf., 110: 508-511. DOI: $10.1115 / 1.3250516$

Wu, S., C.L. Shiu and W.J. Wu, 1996. Analysis on transient heat transfer in annular fins of various shapes with their bases subjected to a heat flux varying as a sinusoidal time function. Comput. Struct., 61: 725-734. DOI: $10.1016 / 0045-7949(96) 00001-6$

Wu, S., C.L. Shiu and W.J. Wu,1996. Analysis on transient heat transfer in annular fins of various shapes with their bases subjected to a heat flux varying as a sinusoidal time function. Comput. Struct., 61: 725-734.

DOI: $10.1016 / 0045-7949(96) 00001-6$

Yang, J.W., C. Scaccia and J. Goodman, 1974. Laminar natural convection about vertical plates with oscillatory surface temperatur. Trans. ASME J. Heat Transf., 96: 9-14. DOI: 10.1115/1.3450149

\section{Nomenclature}

a radius of inner cylinder

$b \quad$ radius of outer cylinder

$A \quad$ steady velocity profile

$B \quad$ periodic velocity profile

$F \quad$ steady temperature profile

$G \quad$ periodic temperature profile

$H$ dimensionless heat generation or absorption parameter

$g \quad$ acceleration due to gravity

$I_{n} \quad$ modified Bessel's function of first kind of order $n$. Where $\mathrm{n}=0,1,2,3, \ldots$

$J_{n} \quad$ Bessel's function of first kind of order $n$. Where $n=0,1,2,3, \ldots$

$K_{n} \quad$ modified Bessel's function of second kind of order $n$. Where $n=0,1,2,3, \ldots$

$M \quad$ dimensionless volume flow rate

$N_{u} \quad$ Nusselt number

$P_{r} \quad$ Prandtl number

$Q_{0} \quad$ dimensional heat generation/absorption parameter

$r \quad$ dimensional radial coordinate

$R \quad$ dimensionless radial coordinate 
St Strouhal number

$t \quad$ time

$T \quad$ temperature of the fluid

$T_{0} \quad$ initial temperature

$T_{1}, T_{2} \quad$ steady temperature at the surfaces of the cylinders

$u \quad$ velocity of fluid

Yn Bessel's function of second kind of order $\mathrm{n}$. Where $n=0,1,2, \ldots$

\section{Greek Alphabets}

a thermal diffusivity of the fluid

$\beta \quad$ coefficient of thermal expansion

$$
\begin{array}{ll}
\tau & \text { radius ratio }\left(\frac{b}{a}\right) \\
\lambda & \text { skin friction } \\
v & \text { kinematic viscosity } \\
\omega & \text { frequency of time-periodic heating }
\end{array}
$$

\section{Subscript}

$1 \quad$ outer surface of inner cylinder

$\lambda \quad$ inner surface of outer cylinder

\section{Appendix}

$I_{0}, I_{1}, K_{0}, K_{1}$ are the modified Bessels of first and second kinds with order zero and one respectively, $C_{1}, C_{2}, C_{3}, C_{4}$ are constants defined as:

$$
\begin{aligned}
& I_{0}(R)=\sum_{k=0}^{\infty} \frac{\left(\frac{R^{2}}{4}\right)^{k}}{(k !)^{2}}, \quad I_{1}(R)=\frac{R}{2} \sum_{k=0}^{\infty} \frac{\left(\frac{R^{2}}{4}\right)^{k}}{k !(k+1) !}, K_{0}(R)=\int_{0}^{\infty} \frac{\cos (R t) d t}{\sqrt{t^{2}+1}}, K_{1}(R)=\frac{2 R \Gamma(1.5)}{\sqrt{\pi}} \int_{0}^{\infty} \frac{\cos (t) d t}{\left(t^{2}+1\right)^{1.5}} \\
& M_{1}=\sqrt{H^{2}-i S t P r}, \quad M_{2}=\sqrt{H^{2}+i S t P r}, \\
& C_{1}=\frac{K_{0}(\lambda H)-K_{0}(H)}{K_{0}(\lambda H) \mathrm{I}_{0}(H)-K_{0}(H)-\mathrm{I}_{0}(\lambda \mathrm{H})}, \quad \mathrm{C}_{2}=\frac{I_{0}(H)-I_{0}(\lambda H)}{K_{0}(\lambda H) I_{0}(H)-K_{0}(H)-I_{0}(\lambda H),}, \quad \mathrm{C}_{4}=\frac{I_{0}(H)-I_{0}(\lambda H)}{K_{0}(\lambda H) I_{0}(H)-K_{0}(H)-I_{0}(\lambda H)} \\
& \mathrm{C}_{3}=\frac{K_{0}(\lambda H)-\mathrm{K}_{0}(H)}{K_{0}(\lambda H) \mathrm{I}_{0}(H)-K_{0}(H)-\mathrm{I}_{0}(\lambda H)}, \quad \mathrm{C}_{6}=\frac{I_{0}\left(M_{2}\right)-I_{0}\left(\lambda M_{2}\right)}{K_{0}\left(\lambda M_{2}\right) I_{0}\left(M_{2}\right)-K_{0}\left(M_{2}\right)-I_{0}\left(\lambda M_{2}\right)} \\
& \mathrm{C}_{5}=\frac{K_{0}\left(\lambda M_{1}\right) I_{0}\left(M_{1}\right)-I_{0}\left(\lambda M_{1}\right)}{\left.K_{0}\left(\lambda M_{2}\right) I_{1}\right)-Y_{0}\left(\lambda M_{1}\right)} \\
& \mathrm{C}_{7}=\frac{Y_{0}\left(M_{2}\right)-K_{0}\left(M_{2}\right)-I_{0}\left(\lambda M_{2}\right)-Y_{0}\left(M_{1}\right)}{Y_{0}\left(\lambda M_{1}\right) I_{0}\left(M_{1}\right)-I_{0}\left(M_{2}\right)-I_{0}\left(\lambda M_{1}\right)}, \quad \\
& \mathrm{C}_{10}=\frac{C_{1} I_{0}(H)+C_{2} K_{0}(H)}{H^{2}}, \mathrm{C}_{9}=\frac{1}{H^{2} I n(\lambda)}\left\{\left[C_{1} I_{0}(\lambda H)+C_{2} K_{0}(\lambda H)\right]-\left[C_{1} I_{0}(H)+C_{2} K_{0}(H)\right]\right\} \\
& \mathrm{C}_{11}=\frac{1}{H^{2} I n(\lambda)}\left\{\left[C_{3} J_{0}(H)+C_{4} Y_{0}(H)\right]-\left[C_{3} J_{0}(\lambda H)+C_{4} Y_{0}(H)\right]\right\}, \quad \mathrm{C}_{12}=-\frac{\left[C_{3} J_{0}(H)+C_{4} Y_{0}(H)\right]}{H^{2}} \\
& \mathrm{C}_{13}=\frac{K_{0}(\sqrt{i S t})-K_{0}(\lambda \sqrt{i S t})}{\left(M_{1}^{2}+i S t\right)\left[K_{0}(\lambda \sqrt{i S t}) i_{0}(\sqrt{i S t})-K_{0}(\sqrt{i S t})-I_{0}(\lambda \sqrt{i S t})\right]}, \\
& \mathrm{C}_{15}=\frac{\frac{K_{0}(\lambda \sqrt{i S t})-K_{0}(\sqrt{i S t})}{\left(M_{1}^{2}+i S t\right)\left[K_{0}(\lambda \sqrt{i S t}) i_{0}(\sqrt{i S t})-K_{0}(\sqrt{i S t})-I_{0}(\lambda \sqrt{i S t})\right]},}{\left(M_{2}^{2}-i S t\right)\left[K_{0}(\lambda \sqrt{i S t}) i_{0}(\sqrt{i S t})-K_{0}(\sqrt{i S t})-I_{0}(\lambda \sqrt{i S t})\right]},
\end{aligned}
$$




$$
\begin{aligned}
& \mathrm{C}_{16}=\frac{I_{0}(\sqrt{i S t})-I_{0}(\lambda \sqrt{i S t})}{\left(M_{1}^{2}-i S t\right)\left[K_{0}(\lambda \sqrt{i S t}) i_{0}(\sqrt{i S t})-K_{0}(\sqrt{i S t})-I_{0}(\lambda \sqrt{i S t})\right]}, \\
& N_{1}=\frac{C_{13}}{\sqrt{i S t}}\left[\lambda I_{1}(\lambda \sqrt{i S t})-I_{1}(\sqrt{i S t})\right]-\frac{C_{14}}{\sqrt{i S t}}\left[\lambda K_{1}(\lambda \sqrt{i S t})-K_{1}(\sqrt{i S t})\right] \\
& N_{2}=\frac{1}{\left(M_{1}^{2}+i S t\right)}\left\{\frac{C_{7}}{M_{1}}\left[\lambda J_{1}\left(\lambda M_{1}\right)-J_{0}\left(M_{1}\right)\right]-\frac{C_{8}}{M_{1}}\left[\lambda Y_{1}\left(\lambda M_{1}\right)-Y_{1}\left(M_{1}\right)\right]\right\} \\
& N_{3}=\frac{C_{15}}{\sqrt{i S t}}\left[\lambda I_{1}(\lambda \sqrt{i S t})-I_{1}(\sqrt{i S t})\right]-\frac{C_{16}}{\sqrt{i S t}}\left[\lambda K_{1}(\lambda \sqrt{i S t})-K_{1}(\sqrt{i S t})\right] \\
& N_{4}=\frac{1}{\left(M_{2}^{2}-i S t\right)}\left\{\frac{C_{5}}{M_{2}}\left[I_{1}\left(M_{1}\right)-\lambda I_{0}\left(\lambda M_{2}\right)\right]-\frac{C_{6}}{M_{2}}\left[K_{1}\left(M_{2}\right)-\lambda K_{0}\left(\lambda M_{2}\right)\right]\right\}
\end{aligned}
$$

